\title{
Research and application of Outdoor parking detection system based on WSN
}

\author{
Yang $\mathrm{Xu}^{1, \mathrm{a}}$,Xiaorong Zhou ${ }^{2, \mathrm{~b}}$ \\ ${ }^{1}$ School of Chongqing university of post and telecommunication, Chongqing 400065, China \\ ${ }^{2}$ School of Chongqing university of post and telecommunication, Chongqing 400065, China \\ axuyang@cqupt.edu.cn, bzhouxr77@foxmail.com
}

Keywords: parking detection, geomagnetic sensor, WSN, low-power.

\begin{abstract}
In order to solve inefficient management and inaccurate hand-timing issues of outdoor parking system, the thesis designs a outdoor parking detection system based on WSN. The system consists of parking terminal node, relay node and master node, the parking terminal node mainly uses STM8L SCM with the geomagnetic sensor and the $433 \mathrm{MHz}$ wireless transceiver module. By wireless communication protocol and low-power design, the system realizes wireless data collection, locating parking space information and automatic timing. Test results show that the system possesses low-power, high-performance benefits, and can actually be applied to outdoor parking spaces detection.
\end{abstract}

\section{Introduction}

In the domestic, Per capita vehicle ownership increases gradually, the demand for parking space is growing, outdoor parking systems begin to derive, especially in the pedestrian street area, build outdoor parking lot at the side of the road by means of parking lots' division, so how to efficiently manage these parking lot has become a serious problem.

At present, the operation mode of domestic outdoor parking is manual times charging, which not only makes parking labor costs increase, but artificial observation and timing is not accurate, these all make the parking lot profit loss. So, looking for a high efficiency, high reliability parking detection system has become an inevitable trend. Considering the efficiency, reliability, low power consumption, low cost, application prospects and other factors, outdoor parking detection system based on WSN can achieve low cost, high reliability and other targets.

\section{System`s basic structure}

Parking detection system consists of three parts: sensor data acquisition parking nodes, network relay nodes and master nodes, parking nodes is responsible for collecting parking geomagnetic information, and transferring the data to the relay node through the CC1101.Relay node is responsible for gathering data, then transferring to the master node. Finally, master node locates parking information and begins to parking timing while receiving data. The overall structure of the system is shown in Fig.1. 


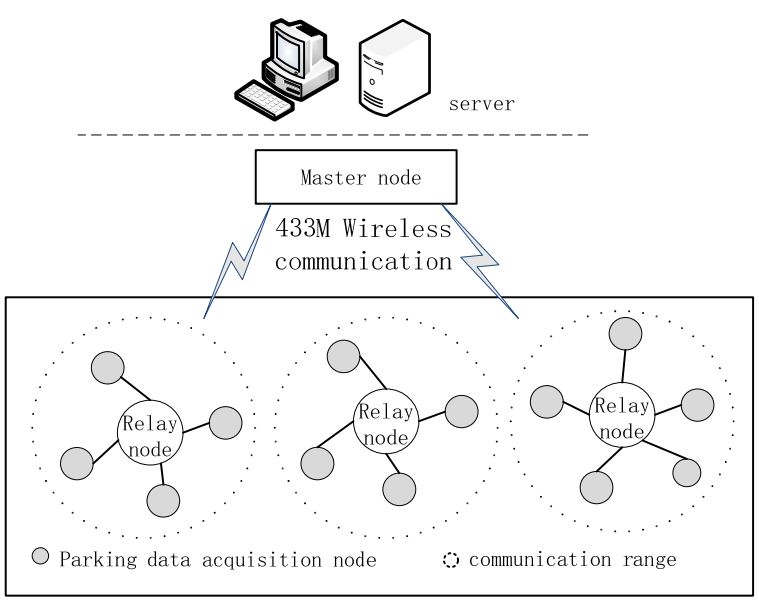

Fig.1 system structure

\section{Parking detection node design}

\subsection{Hardware design}

The Ultralow power STM8L series is STMicroelectronics STM8 CPU core 8-bit microcontrollers, providing high performance and ultralow power consumption benefits, STM8L151 is applied in the design.

CC1101 is a low-cost and low-power wireless transceiver produced by TI company, it provides efficient SPI interface, frequency bands is $387-464 \mathrm{MHZ}$, can avoid the $2.4 \mathrm{GHZ}$ signal interference, improving the reliability of wireless communication effectively. The chip work frequency is 433 MHZ in the design.

Freescale's MAG3110 is a small, low-power digital three-axis magnetometer, it uses a standard $\mathrm{I} 2 \mathrm{C}$ interface, and data output rate is $80 \mathrm{HZ}$.

The hardware architecture of the system is shown in Fig. 2.

\subsection{Software Design}

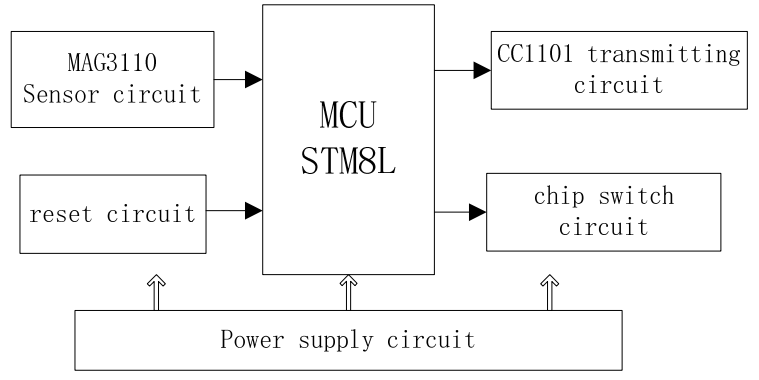

Fig. 2 system hardware architecture

Geomagnetic information of parking spaces are different, so the data calibration is necessary. Calibration Uses the average median filtering method can avoid impulse interference. After the node power on, acquisition 100 groups of data, remove the maximum and minimum value and then averaged, the calibration values is obtained. Then the node begins the normal data acquisition cycle.

According to the vehicle parked frequency at the parking spaces to set the node sleeping cycle, nodes periodically wake up from hibernation and collect parking geomagnetic data 5 times, through the threshold detection to judge whether the vehicle is parked into the parking space, if not, then the node goes back to sleep and waits for the next waking up again, if all data judgment exceed the threshold, it indicating the parking space exists vehicle, then wake up wireless module and begin wireless data transmission, data processing and parking timing will be done at the host. Parking detection process is shown in Fig. 3. 


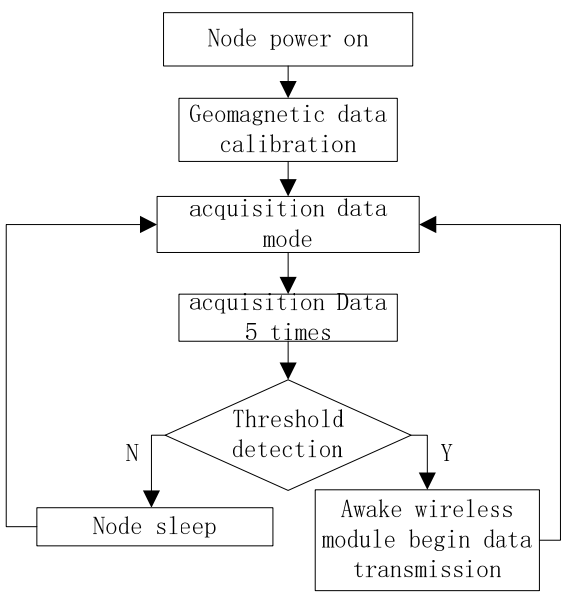

Fig. 3 Parking detection process

\section{Communication Protocol}

\subsection{Protocol process}

Due to the different size of the outdoor parking lot and ordinary parking lot, outdoor parking spaces are often scattered, relatively small number of parking spaces within a communication range, so the protocol architecture adopts a tree structure, the bottom are the data acquisition node, a certain number of bottom nodes consist a tree branch, data flow to the relay node, and then flow to the master node similarly.

\subsection{Data frame format}

Data Frame: frame for transmission of data packets, mainly transmitted parking geomagnetic data.

Response frame: frame for acknowledging receipt of success, to ensure the reliability of the communication link.

Command frame: frame for transmitting control commands.

Forwards frames: frame for receiving parking data at relay node and forwarding if it correctly.

\section{5. low-power design}

\section{1 parking node low-power design}

Owing to the terminal node is in the outdoor, powered by batteries, so it is necessary to consider power consumption. The current of a node always in active working state can reach $25 \mathrm{~mA}$, it can only maintain a few weeks for ordinary battery without low-power design, which cannot meet the power requirements apparently, so it needs for low-power design.

STM8L151 MCU has five low-power features, in practical applications, the microcontroller is set to active halt mode, the wireless module is set to sleeping mode, the sensor is set to power off state, set the MCU sleeping cycle as 3s, set the data acquisition time of sensor as $25 \mathrm{~ms} / \mathrm{time}$, collecting data five times in total $125 \mathrm{~ms}$, after data threshold detection to determine whether to upload the data. In the whole work process, wireless module transmits data only when the car stopped in and drive out, the rest periods node are in dormant. The current of sensor node is about $5 \mathrm{~mA}$, so all current consumption in a period is

$$
5 \times 4.17 \%+100 \times 10^{-3} \times 95.83 \%=304.33 \mathrm{uA}
$$

Ordinary $3000 \mathrm{mAh}$ battery can maintain

$$
3000 \div 0.30433 \div 24=410 \text { days }
$$

It can suffice parking node power requirements. Parking node low power consumption patterns process is shown in Fig. 4. 


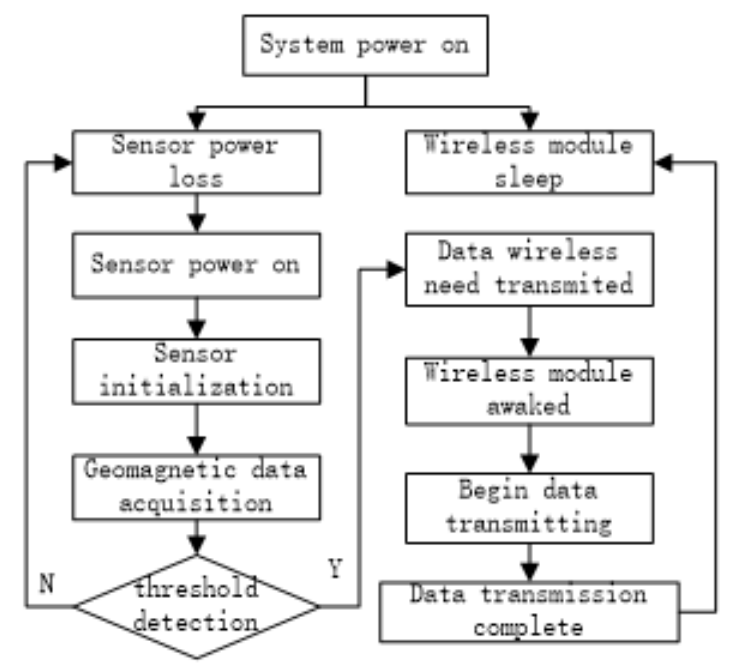

\section{2 relay node low power design}

Fig. 4 Parking node low power consumption patterns process

The main function of relay node is collecting different network data then transfer to the master node, when parking node begin wireless data sending, relay node is awakened into receiving state.

CC1101 support WOR feature, which allows the module to covert between sleeping state and operating state, when the data rate is $100 \mathrm{kbps}$, a complete data packet transmission time is $16 \mathrm{x} 8$ / $100=1.28 \mathrm{~ms}$, so five packet transmission time need $6.4 \mathrm{~ms}$, set the duty cycle to $1.563 \%$, the whole WOR period is $\quad 6.4 \div 1.563 \%=409.47 \mathrm{~ms}$. Calculation of a cycle of current consumption is

$3000 \mathrm{mAh}$ battery can maintain

$$
5 \times 10^{-3} \times 98.437 \%+20 \times 1.563 \%=317.52 \mathrm{uA}
$$

$$
3000 \div 0.31752 \div 24=394 \text { days }
$$

Relay node working state transformation is shown in Fig. 5.

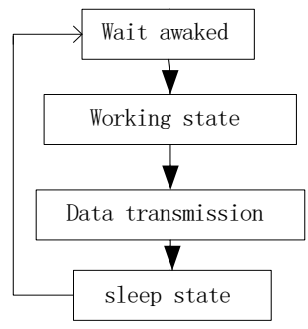

Fig. 5 Relay node working state transformation

\section{Experiment results}

Experimental procedure and conditions: Install node in the middle position of parking spaces, the node begins data calibration to stable, after a period of time, the vehicle parks in, then vehicle drives out, the experiment progress end. Experimental measured three axis geomagnetic data as shown in Fig. 6.

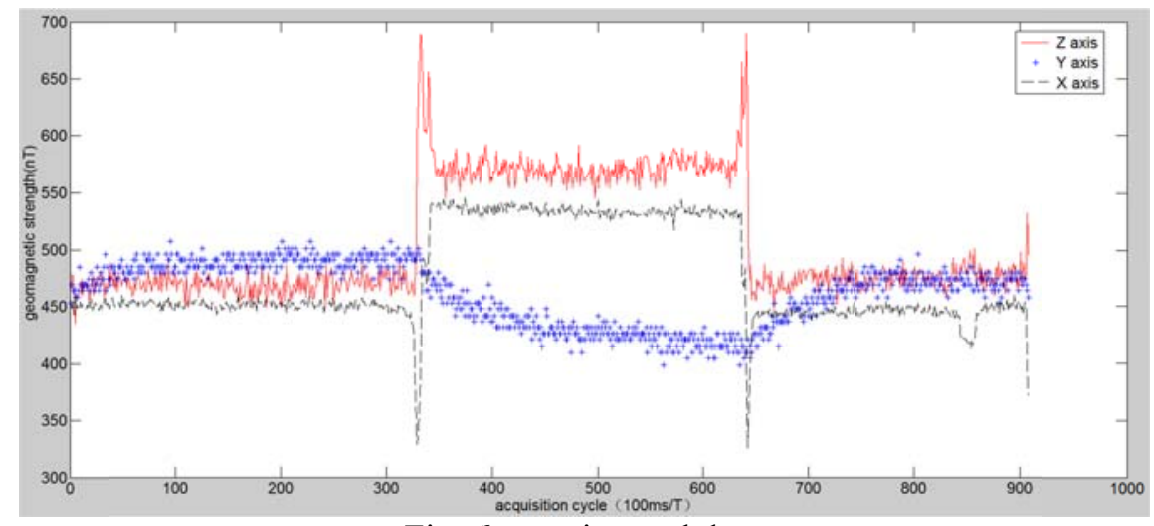

Fig. 6 experimental data

From Figure 6, when there is no vehicle in parking space, geomagnetic data is normal, when 
the vehicle parks in and drives out, the vehicle cuts magnetic field lines, geomagnetic data changes significantly, so it can clear judge the parking space state and begin timing. In the figure, the $\mathrm{x}$ axis and $\mathrm{z}$ axis data changes significantly, so the two-axis data can be used to threshold detection.

In reality, there exists different type of vehicles, selecting the three type of vehicle to conduct the experiment, testing mini vehicle, common vehicle and SUVs respectively, experiment data is obtained and the data comparison is shown in Fig. 7.

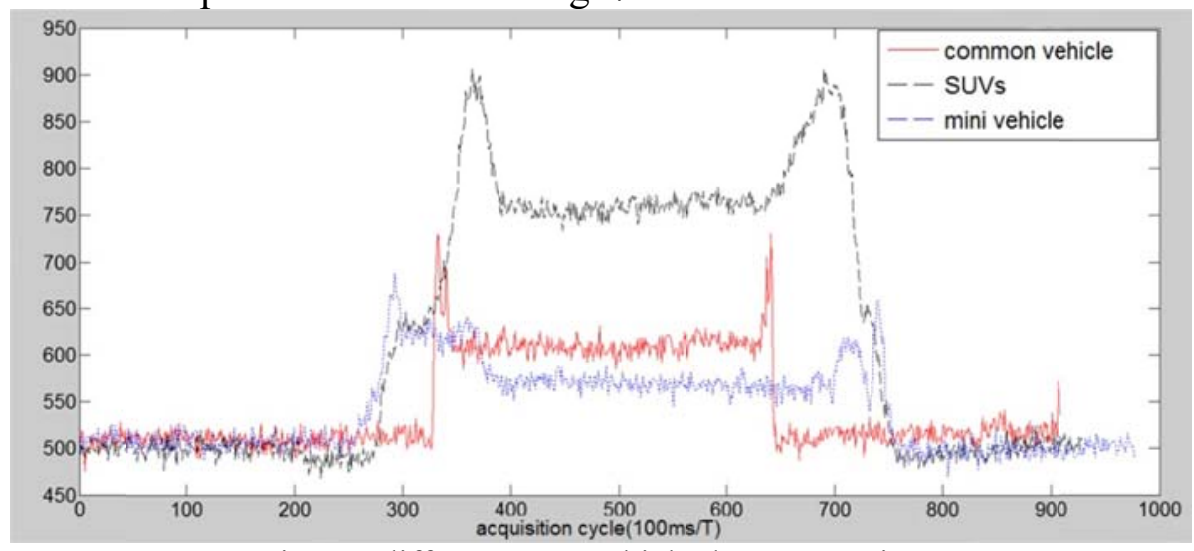

Fig. 7 different type vehicle data comparison

From the test data of figure 7, the data amplitude of different vehicles is different, the small vehicle data changes significantly lower than large vehicle, it shows that the effects of the geomagnetic data is directly related to vehicle size. The change of geomagnetic data all above 100 $\mathrm{nT}$, so the threshold can be set as 70nT, it avoids misjudgment for small vehicles and the interference of external factors. It verifies the whole system reliability.

\section{Conclusion}

Parking detection system use STM8L151 microcomputer with CC1101 chip and MAG3110 geomagnetic sensor, and bases on the $433 \mathrm{MHZ}$ wireless communication protocol design, achieving parking data acquisition and records. The system reduces labor costs and power consumption, while improves work efficiency, so the whole system meets the low power, low cost and high reliability requirements.

\section{References}

[1] J. Yang, J. Portilla,T. Riesgo. Smart parking service based on Wireless Sensor Networks. 2012, 2(1):6029-6034.

[2] D. Xin. Street Light Monitoring and Energy-saving System Based On CC1101 Wireless Ad Hoc Network. Automation \& Instrumentation, 2011, 26(2):20-23.

[3] Z. Min-hua, L. Li, H. Na. Design of Water Quality Monitoring System Based on Wireless Sensor Network.Computer Engineering. 2014, 29(2):92-96.

[4] W. Xing-fu, J. Na, M. Xu-fei. Parking Guidance and Information System Based on Wireless Magnetic Node. Journal of Chinese Computer Systems. 2015, (09):2031-2036. 